\title{
The Relation between Ambient Temperature and Asthma Exacerbation in Children: A Systematic Review
}

\author{
Hamid Reza Shoraka ${ }^{1,3}$, Moslem Taheri Soodejani', Omid Abobakri', Narges Khanjani'* \\ 'Dept. of Epidemiology and Biostatistics, Kerman University of Medical Sciences, Kerman, Iran \\ ${ }^{2}$ Environmental Health Engineering Research Center, Kerman University of Medical Sciences, Kerman, Iran \\ ${ }^{3}$ Vector-borne Diseases Research Center, North Khorasan University of Medical Sciences, Bojnurd, Iran
}

Article Info

\section{Article Notes}

Received: November 26, 2018

Accepted: January 04, 2019

\section{${ }^{*}$ Correspondence:}

Dr. Narges Khanjani, Environmental Health Engineering Research Center, Kerman University of Medical Sciences,

Kerman, Iran; Telephone No: 034-3132-5102;

Email: $n \_k h a n j a n i @ k m u . a c . i r$

C 2019 Khanjani N. This article is distributed under the terms of the Creative Commons Attribution 4.0 International License.

\section{Keywords}

Ambient Temperature

Asthma Exacerbation

Children

Systematic Review

Airway

\section{Abstract}

Background: Asthma is one of the most common chronic noncommunicable diseases which is seen more in the developed than developing countries of the world. Recurrence and exacerbations of the disease are common among patients and often lead to hospitalization and therapeutic interventions. Ambient air temperature might be related to the relapse of asthma. This review was conducted to investigate the relation between ambient temperature and exacerbations of asthma in children.

Methods: Related articles were searched in PubMed, Web of Science, Science Direct, and Scopus databases with appropriate keywords and no specific limitation on October 1,2018 . Initially, the relevance of the articles was examined using the title and abstract. Out of 2633 articles, 23 articles were eligible according to the inclusion and exclusion criteria.

Results: Fourteen studies had reported inverse relations; and showed as the temperature dropped, the number of asthma attacks increased in children. Nine papers observed a relation between hot weather and asthma attacks, 3 studies reported a relation between temperature differences and asthma attacks, and two studies did not show any relation. Some studies suggested the increased incidence of asthma in the 5-14 year old age group was associated with the start of the school year and probably due to the spread of viral diseases, not temperature changes.

Conclusion: Extreme temperatures are likely to cause exacerbation of childhood asthma.

\section{Introduction}

Asthma is one of the most common chronic airway and non-communicable diseases worldwide and its prevalence has increased in recent years ${ }^{1}$. This disease is characterized by chronic inflammation and obstruction of the airways. According to the WHO report, 235 million people suffer from this disease ${ }^{2}$.

Recurrence and exacerbations of this disease are common among patients, which often leads to hospitalization and therapeutic interventions for improving lung function ${ }^{3}$. Several studies have been conducted to investigate the relation between air pollutants such as ozone and fine particulate matter and the progression and exacerbations of asthma. There is also evidence that exposure to environmental factors such as ambient temperature is associated with recurrence of asthma ${ }^{4}$, and even deterioration and death due to respiratory failure ${ }^{5,6}$.

Average global temperature has increased $0.85 \circ \mathrm{C}$, between 
1880 and $2012^{7}$. Nowadays all world countries are affected by global warming and its effect is not limited to a specific region of the world ${ }^{8}$.

Air temperature may directly or indirectly be related to the recurrence of asthma. Cold air may directly affect airways and cause hyper-responsiveness. It might also indirectly trigger asthma by predisposing people to viral diseases, or air pollution. Air conditioners used in warm weather may also trigger the recurrence of asthma ${ }^{9}$. Several studies have been conducted in different countries to investigate the relation between ambient temperature variations and the incidence and/or recurrence of asthma. Studies have reported clusters of asthma recurrence and hospitalization in some seasons ${ }^{10}$. Children are one of the sensitive groups affected by environmental factors. These factors might cause asthma recurrence and hospitalization in sensitive children ${ }^{11}$.

Understanding the environmental factors that trigger asthma recurrence and hospitalization in children can help prevent these attacks by modifying lifestyle or reducing exposure to these environmental factors ${ }^{12}$. Various studies have reported different results about the role of ambient temperature on the occurrence of asthma. These studies have been conducted in different geographic, social and racial regions and have examined the effect of low or high temperatures, or heat/cold waves, on the exacerbation of asthma ${ }^{13,14}$. These studies have reported various associations and it is necessary to provide an overall conclusion from these studies to help public health policymaking. Although a systematic review was recently conducted about ambient temperature and childhood asthma by $\mathrm{Xu}$ et $\mathrm{al}^{15}$, but this review did not include some articles ${ }^{4,16-29}$.

This review study was conducted to investigate the relation between ambient temperature and asthma focusing on recurrence and exacerbation of the disease in children.

\section{Materials \& Methods}

\section{Inclusion Criteria}

Studies were included in this review if they had considered at least one temperature index as an exposure, had evaluated the age group under 17 years separately, the outcome was investigated in humans, and recurrence or hospitalization due to exacerbation of asthma was reported as a consequence. Also, papers were included which had included at least one year data.

\section{Search Strategy}

PubMed, Web of Science, Science Direct, and Scopus databases were searched for relevant articles. No time limit was implemented.
The keywords "temperature", "weather", "climate"," heat", "hot", "cold" and "ambient temperature" were used as exposure terms and "hospital*","admission*", "emergence*", "exacerbation" and "asthma" were used as outcome terms. The final search was done on October 1, 2018. In order to retrieve all related articles which included various age groups, we did not use "Children" as a keyword. Hence, we screened full-text articles to see if they had included children as a separate category.

\section{Study selection}

After searching different databases, the titles and abstracts of the retrieved articles were imported into EndNote software and duplicates were removed based on title, author and year of publication.

Initially, the relevance of the articles was examined based on title and abstract. Out of 2633 articles, 88 were selected for full-text evaluation. The full-text articles were evaluated using the inclusion and exclusion criteria.

The author's name, study period, population size, type of study, type of exposure variable and outcome, statistical analysis, and the main results were extracted from the finally selected articles.

Due to the differences in study methodologies, statistical analysis, and different temperature indices, conducting a meta-analysis was not possible.

\section{Result}

After screening through several steps, shown in Figure 1, 23 articles were selected for the review.

The characteristics of these articles are summarized in Table 1. The studies were from different parts of the world including Europe, America and Asia, but no study was conducted in Africa. The studies had been conducted in 12 countries.

In order to further examine the geographical distribution and the characteristics of the area in which the studies were conducted, the Köppen-Geiger climate classification was used, which includes 5 climate zones (figure 2). Three of the studies had been done in the equatorial region ${ }^{17,21,22}$. There were no studies from the arid and polar regions. The

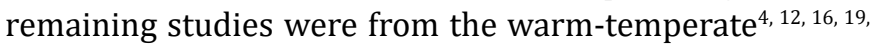
$20,23-26,28-35$ and snow regions ${ }^{18,27,36}$.

The studies included between one to nineteen years data. Studies had used different variables for measuring ambient temperature. Daily minimum, maximum and mean temperatures were the most common variables used in 16 studies $^{4,12,16-19,21,22,24,25,28,29,31,32,34-36}$ followed by monthly minimum, maximum and mean temperature in 5 studies $^{20,}$ $23,26,30,33$ and one article used hourly meteorological data ${ }^{27}$.

Different definitions had been used to define the 


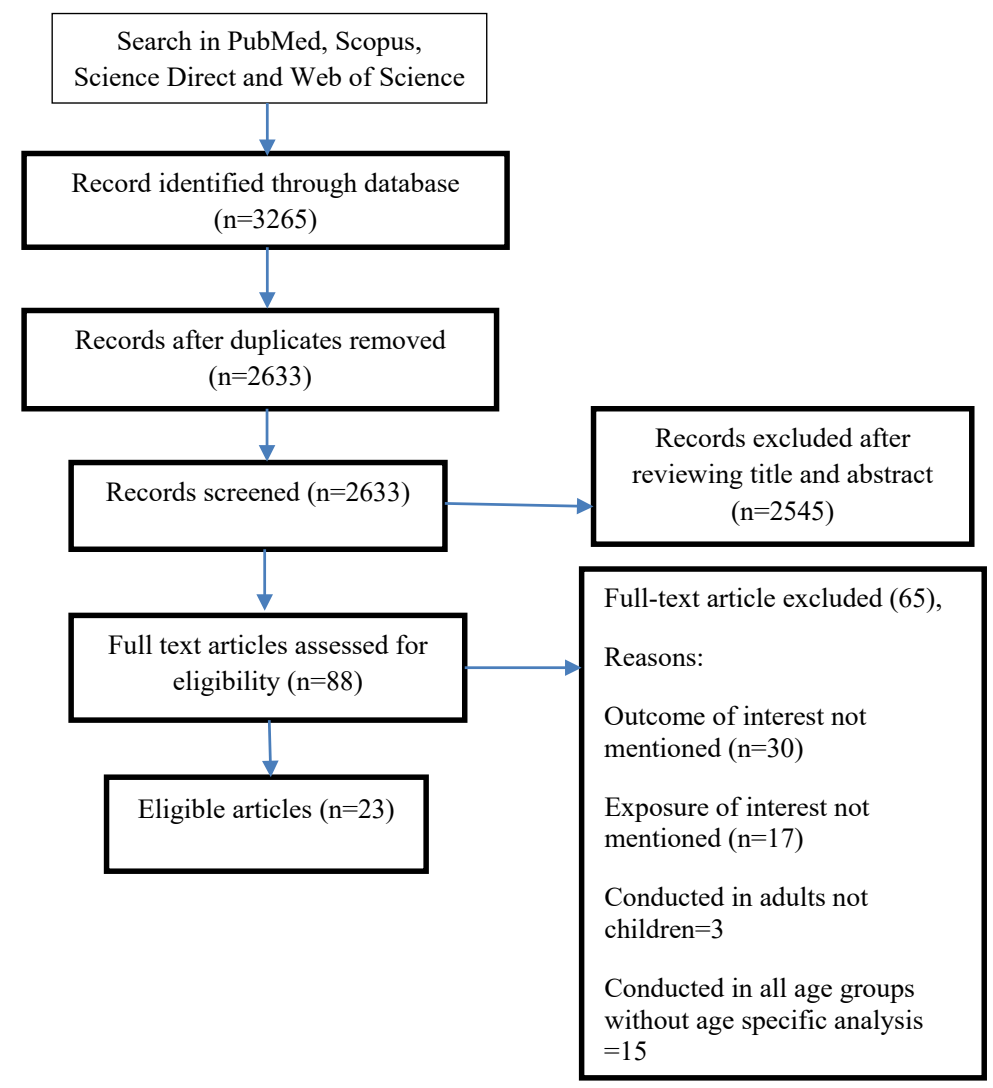

Figure 1: Search results and study selection

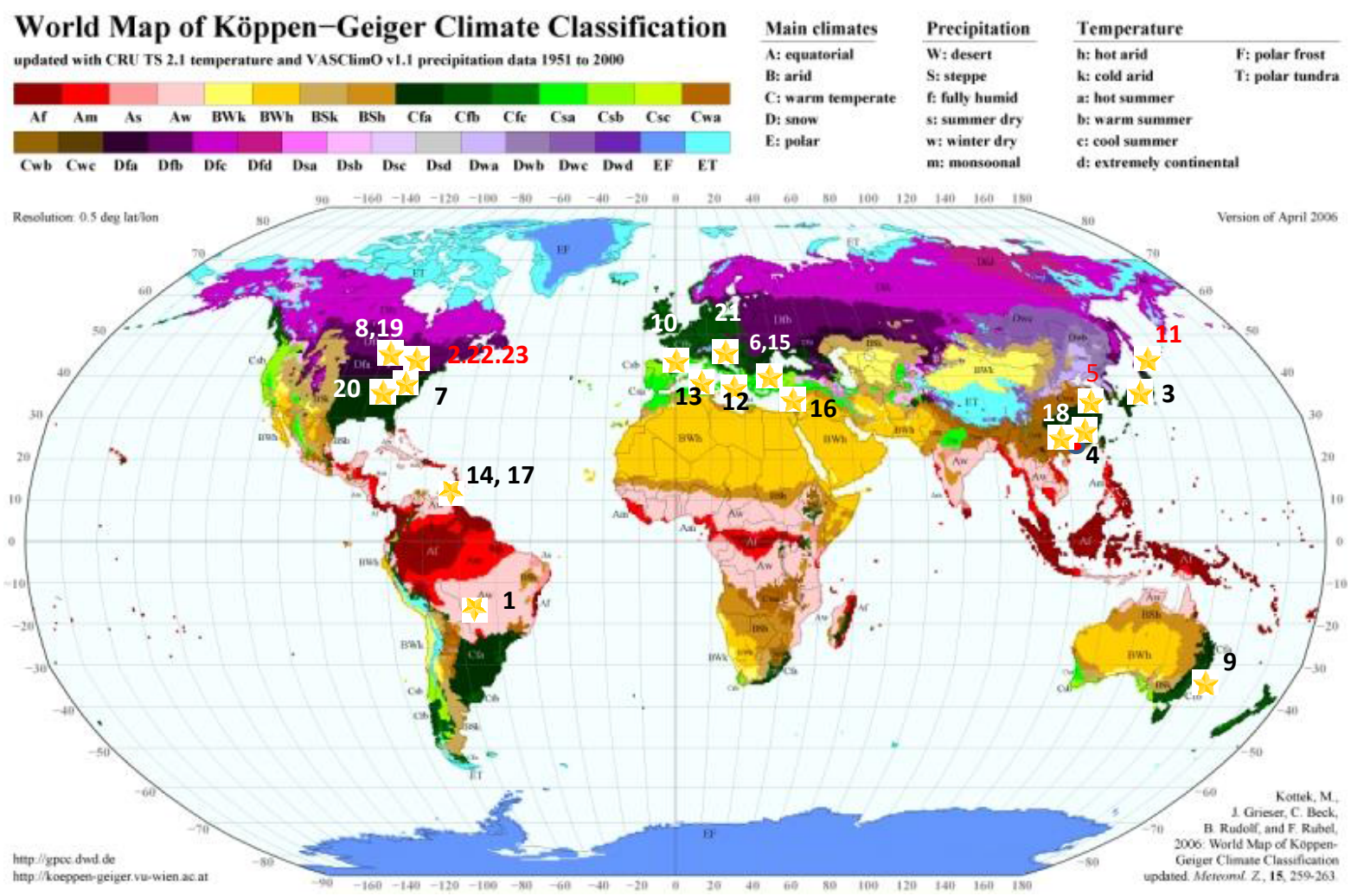

Figure 2: Distribution of studies across five Köppen Geiger climate zones (A-E). Study numbers on the map are defined in Table 1. 
Table 1. Summary of the studies included in this systematic review

\begin{tabular}{|c|c|c|c|c|c|c|c|c|c|c|c|c|c|}
\hline ID & $\begin{array}{l}\text { First Author } \\
\text { Year, location }\end{array}$ & $\begin{array}{l}\text { Population } \\
\text { size }\end{array}$ & $\begin{array}{l}\text { Study } \\
\text { period }\end{array}$ & Ages & $\begin{array}{l}\text { Outcome } \\
\text { definition }\end{array}$ & $\begin{array}{l}\text { Exposure } \\
\text { definition }\end{array}$ & Study type & $\begin{array}{l}\text { Köppen- } \\
\text { Geiger Climate } \\
\text { Classification }\end{array}$ & $\begin{array}{l}\text { Dominant } \\
\text { gender }\end{array}$ & Lag time & $\begin{array}{l}\text { Other variables } \\
\text { included }\end{array}$ & $\begin{array}{l}\text { Statistical } \\
\text { analysis }\end{array}$ & Main results \\
\hline 1 & $\begin{array}{l}\text { De Souza, } \\
\text { 2015, } \\
\text { Brazil(17) }\end{array}$ & 5844 & $\begin{array}{l}2008- \\
2010\end{array}$ & $<9$ & ICD10-(J45) & $\begin{array}{l}\text { Daily Mean } \\
\text { Temperature } \\
\text { (DMT) }\end{array}$ & Ecologic & A & $\mathrm{NR} ?$ & $0-30$ & $\begin{array}{c}\text { Humidity, Rain } \\
\text { fall, Wind Speed, } \\
\mathrm{O}_{3}\end{array}$ & $\begin{array}{l}\text { Pearson } \\
\text { Correlatio }\end{array}$ & $\begin{array}{l}\text { Daily hospital admissions showed } \\
\text { significant negative correlations with } \\
\text { DMT ( } r=-0.214, P<0.001)\end{array}$ \\
\hline 2 & $\begin{array}{c}\text { Fitzgerald, } \\
2014, \text { USA(4) }\end{array}$ & 396043 & $\begin{array}{l}1991- \\
2007\end{array}$ & $<17$ & ICD9-(493.00) & $\begin{array}{l}\text { Daily Mean } \\
\text { Temperature }\end{array}$ & Ecologic & C & Female & $0-4$ & PM2.5-Sex-Race & GAM & $\begin{array}{l}\text { Decrease in admissions during a cold } \\
\text { spell in the winter months for all } \\
\text { subgroups. A mean decline of } 4.9 \\
\% \text { in asthma admissions happened } \\
\text { statewide ( } 95 \% \mathrm{Cl}-7.8,-1.9 \%) \text {. }\end{array}$ \\
\hline 3 & $\begin{array}{l}\text { Hashimoto, } \\
\text { 2004, } \\
\text { Japan(12) }\end{array}$ & 5559 & $\begin{array}{l}1998- \\
2002\end{array}$ & $2-15$ & $\begin{array}{l}\text { Physical findings } \\
\text { such as dyspnea } \\
\text { with wheezes }\end{array}$ & $\begin{array}{l}\text { Daily Min, Max } \\
\text { and Mean } \\
\text { Temperature }\end{array}$ & Ecologic & C & NR & 3 & $\begin{array}{c}\text { Barometric } \\
\text { Pressure, } \\
\text { Humidity/Vapor } \\
\text { Pressure, Wind } \\
\quad \text { Speed }\end{array}$ & $\begin{array}{l}\text { Logistic } \\
\text { Regression }\end{array}$ & $\begin{array}{l}\text { The rapid decrease of temperature } \\
\text { within a 3-day period can increase } \\
\text { the risk of asthma attacks. } \beta=0.05 \text {, } \\
\qquad \mathrm{Cl}=0.02-0.07\end{array}$ \\
\hline 4 & $\begin{array}{l}\text { Lam, 2016, } \\
\text { Hong } \\
\text { Kong(31) }\end{array}$ & 2402 & $\begin{array}{l}2004- \\
2011\end{array}$ & $<15$ & ICD9-(493.00) & $\begin{array}{l}\text { Daily Mean } \\
\text { Temperature }\end{array}$ & Time -Series & C & NR & $20-30$ & $\begin{array}{l}\text { Air Pollutants, } \\
\text { Solar Radiation, } \\
\text { Wind Speed }\end{array}$ & GAM, DLNM & $\begin{array}{c}\text { Asthma admissions increased at } \\
\text { high temperatures in the hot season } \\
\text { and at low temperatures in the cold } \\
\text { season } R R=1.19\end{array}$ \\
\hline 5 & $\begin{array}{l}\text { Liu,2007, } \\
\text { China(23) }\end{array}$ & 445 & $\begin{array}{l}2000- \\
2004\end{array}$ & $0-14$ & $\begin{array}{l}\text { Diagnosis by } \\
\text { clinicians }\end{array}$ & $\begin{array}{l}\text { Monthly Mean } \\
\text { Temperature }\end{array}$ & $\begin{array}{l}\text { Historical } \\
\text { Cohort }\end{array}$ & C & Male & NR & $\begin{array}{c}\text { Air Pressure, } \\
\text { Relative Humidity, } \\
\text { Wind Speed }\end{array}$ & $\begin{array}{l}\text { Linear } \\
\text { Regression } \\
\text { Analysis }\end{array}$ & $\begin{array}{c}\text { Higher incidence was related with } \\
\text { lower temperature, }(r=-0.320, P< \\
0.05)\end{array}$ \\
\hline 6 & $\begin{array}{c}\text { Nastos,2006, } \\
\text { Greece(24) }\end{array}$ & 2764 & $\begin{array}{l}2001- \\
2003\end{array}$ & $0-14$ & $\begin{array}{l}\text { Admitted with } \\
\text { the diagnosis } \\
\text { of "asthma", } \\
\text { "asthmatic } \\
\text { bronchitis" } \\
\text { or "wheezy } \\
\text { bronchitis" }\end{array}$ & $\begin{array}{l}\text { Daily Min, Max } \\
\text { and Mean } \\
\text { Temperature, } \\
\text { Diurnal } \\
\text { Temperature }\end{array}$ & Time-Series & C & Male & NR & $\begin{array}{l}\text { Water Vapor } \\
\text { Pressure and Cold } \\
\text { Anti Cyclonic }\end{array}$ & $\begin{array}{c}\text { Pearson } \\
\text { Correlation }\end{array}$ & $\begin{array}{l}\text { Low temperature, significantly } \\
\text { correlated with an increase in the } \\
\text { number of asthma admissions }\end{array}$ \\
\hline 7 & $\begin{array}{c}\text { Soneja,2016, } \\
\text { USA(34) }\end{array}$ & 119523 & $\begin{array}{l}2000- \\
2012\end{array}$ & $<17$ & ICD9-(493.00) & $\begin{array}{l}\text { Daily Max } \\
\text { Temperature, } \\
\text { Extreme Heat }\end{array}$ & Case-Crossover & C & Female & $0-2$ & Sex, Race & $\begin{array}{l}\text { Conditional } \\
\text { Logistic } \\
\text { Regression }\end{array}$ & $\begin{array}{c}\text { Exposure to extreme heat events } \\
\text { was associated with a } 3 \% \text { increase } \\
\text { in the risk of hospital admission for } \\
\text { asthma (Odds Ratio: } 1.03 \text { ) }\end{array}$ \\
\hline
\end{tabular}




\begin{tabular}{|c|c|c|c|c|c|c|c|c|c|c|c|c|c|}
\hline 8 & $\begin{array}{l}\text { Wasilevich, } \\
\text { 2012, } \\
\text { USA(27) }\end{array}$ & 4804 & $\begin{array}{l}2000- \\
2001\end{array}$ & 3-18 & $\begin{array}{l}\text { ICD9-(493.00- } \\
493.99\end{array}$ & $\begin{array}{l}\text { Hourly Min, } \\
\text { Max and Mean } \\
\text { Temperature }\end{array}$ & Case-Crossover & $\mathrm{D}$ & Male & NR & $\begin{array}{l}\text { Relative Humidity, } \\
\text { Barometric } \\
\text { Pressure, } \\
\text { Wind Speed, } \\
\text { Thunderstorm } \\
\text { Activity and Air } \\
\text { Pollutant } \\
\end{array}$ & $\begin{array}{l}\text { Conditional } \\
\text { Logistic } \\
\text { Regression }\end{array}$ & $\begin{array}{l}\text { The case crossover study showed } \\
\text { a statistically significant inverse } \\
\text { relation between ED visits and } \\
\text { maximum } 24 \text {-hour temperature } \\
\text { change after adjustment for climatic } \\
\text { factors, } R R=0.972, P=0.01\end{array}$ \\
\hline 9 & $\begin{array}{c}\text { Xu,2013, } \\
\text { Australia(35) }\end{array}$ & 13324 & $\begin{array}{l}2003- \\
2009\end{array}$ & $0-14$ & ICD10-(J45) & $\begin{array}{l}\text { Daily Min , } \\
\text { Max and Mean } \\
\text { Temperature }\end{array}$ & Ecologic & C & Male & $\begin{array}{c}0-1,0-13 \\
0-21\end{array}$ & PM10, 03 & $\begin{array}{l}\text { Poisson } \\
\text { Linear } \\
\text { Regression }\end{array}$ & $\begin{array}{c}\text { Male children and children aged } 0-4 \\
\text { years were particularly sensitive to } \\
\text { hot temperature ( } \mathrm{RR}=1.61 \mathrm{Cl}=1.22 \\
\text { to } 2.14 \text { ), and children aged } 10-14 \\
\text { years were particularly sensitive to } \\
\text { cold temperatures ( } \mathrm{RR}=1.96, \mathrm{Cl}=1.03 \\
\text { to } 3.50)\end{array}$ \\
\hline 10 & $\begin{array}{l}\text { Altzibar, } \\
\text { 2015, Spain } \\
\text { (16) }\end{array}$ & 59500 & $\begin{array}{l}2004- \\
2009\end{array}$ & $<15$ & ICD9-(493.00) & $\begin{array}{l}\text { Daily Min , } \\
\text { Max and Mean } \\
\text { Temperature }\end{array}$ & Ecologic & C & Male & NR & $\begin{array}{c}\text { Age, Sex, Air } \\
\text { Pollutant, Relative } \\
\text { Humidity and Flu } \\
\text { Status }\end{array}$ & $\begin{array}{l}\text { Pearson } \\
\text { Correlation }\end{array}$ & $\begin{array}{c}\text { Asthma exacerbations were } \\
\text { correlated negatively with } \\
\text { temperature. }(r=-0.485 \mathrm{Cl}=-0.543 ;- \\
0.423)\end{array}$ \\
\hline 11 & $\begin{array}{l}\text { Ehara,2000, } \\
\text { Japan(18) }\end{array}$ & 205 & 1997 & $1-16$ & $\begin{array}{l}\text { Asthmatic } \\
\text { symptoms, such } \\
\text { as wheezing and } \\
\text { expiratory stridor }\end{array}$ & $\begin{array}{l}\text { Daily Diurnal } \\
\text { Temperature }\end{array}$ & Ecologic & $\mathrm{D}$ & Male & 1 & $\begin{array}{c}\text { Barometric } \\
\text { Pressure and } \\
\text { relative Humidity }\end{array}$ & $\begin{array}{l}\text { Mann- } \\
\text { Whitney } \\
\text { U-test }\end{array}$ & $\begin{array}{c}\text { Diurnal difference between } \\
\text { maximum and minimum } \\
\text { temperatures of } 1 \text { day before } \\
\text { admissions was larger than that of } 1 \\
\text { day before days with no admissions } \\
\mathrm{P}=0.02\end{array}$ \\
\hline 12 & $\begin{array}{l}\text { Grech,2002, } \\
\text { Malta(20) }\end{array}$ & 2916 & $\begin{array}{l}1994- \\
1998\end{array}$ & $0-59$ & $\begin{array}{l}\text { Diagnosis } \\
\text { of asthma } \\
\text { or wheezy } \\
\text { bronchitis }\end{array}$ & $\begin{array}{l}\text { Monthly Min , } \\
\text { Max and Mean } \\
\text { Temperature }\end{array}$ & Ecologic & C & NR & NR & Age Group & $\begin{array}{l}\text { Spearman } \\
\text { Correlation }\end{array}$ & $\begin{array}{l}\text { Ranked mean monthly ambient } \\
\text { temperatures correlated significantly } \\
\text { with monthly admissions in the } \\
\text { pediatric cohort }(r=-0.71, p<0.0001)\end{array}$ \\
\hline 13 & $\begin{array}{l}\text { Hervas,2015, } \\
\text { Spain(30) }\end{array}$ & 371 & $\begin{array}{l}2007- \\
2011\end{array}$ & 5-14 & $\begin{array}{l}\text { ICD9-(493.0- } \\
493.9)\end{array}$ & $\begin{array}{l}\text { Monthly Min, } \\
\text { Max and Mean } \\
\text { Temperature }\end{array}$ & $\begin{array}{l}\text { Longitudinal } \\
\text { Retrospective }\end{array}$ & C & Male & $30-60$ & $\begin{array}{c}\text { Water Vapor } \\
\text { Pressure, Relative } \\
\text { Humidity }\end{array}$ & $\begin{array}{l}\text { Multivariate } \\
\quad \text { linear } \\
\text { Regression }\end{array}$ & $\begin{array}{l}\text { The Regression equation showed } \\
\text { a } 7.3 \% \text { increase in the number of } \\
\text { monthly asthma exacerbations } \\
\text { for each degree decrease in } \\
\text { temperature. }(\beta=27.2, P<0.0001)\end{array}$ \\
\hline 14 & $\begin{array}{l}\text { Ivey,2003, } \\
\text { Trinidad and } \\
\text { Tobago(22) }\end{array}$ & 45842 & $\begin{array}{l}1997- \\
1999\end{array}$ & $0-65$ & $\begin{array}{l}\text { Diagnosis of } \\
\text { acute asthma } \\
\text { and received } \\
\text { bronchodilator } \\
\text { nebulization }\end{array}$ & $\begin{array}{l}\text { Daily Min } \\
\text { and Diurnal } \\
\text { Temperature }\end{array}$ & Ecologic & A & NR & 1 & $\begin{array}{c}\text { Relative Humidity, } \\
\text {, Barometric } \\
\text { Pressure, Wind } \\
\text { Speeds }\end{array}$ & $\begin{array}{l}\text { Multiple } \\
\text { Regression }\end{array}$ & $\begin{array}{l}\text { Results of multiple regression } \\
\text { indicated that temperature } \\
\text { difference }(P<0.001) \text {, and minimum } \\
\text { temperature }(P<0.001) \text { were } \\
\text { predictors of pediatric visits }\end{array}$ \\
\hline 15 & $\begin{array}{c}\text { Nastos,2008, } \\
\text { Greece(33) }\end{array}$ & NA & $\begin{array}{l}1978- \\
2000\end{array}$ & $0-14$ & $\begin{array}{l}\text { Diagnosis of } \\
\text { "asthma," } \\
\text { "asthmatic } \\
\text { bronchitis" } \\
\text { or "wheezy } \\
\text { bronchitis," }\end{array}$ & $\begin{array}{l}\text { Monthly Diurnal } \\
\text { Temperature }\end{array}$ & Ecologic & C & NR & $0-3$ & $\begin{array}{c}\text { Relative Humidity, } \\
\text { Absolute } \\
\text { Humidity and } \\
\text { Wind Speed }\end{array}$ & $\begin{array}{l}\text { Generalized } \\
\text { Linear } \\
\text { Models }\end{array}$ & $\begin{array}{c}\text { There was a negative relation mean } \\
\text { monthly air temperature and asthma } \\
\text { admissions in the age group } 0-4 \\
\text { years }(\mathrm{B}=-0.0376, \mathrm{P}=<0.0001 \text { ) }\end{array}$ \\
\hline
\end{tabular}




\begin{tabular}{|c|c|c|c|c|c|c|c|c|c|c|c|c|c|}
\hline 16 & $\begin{array}{c}\text { Garty,1998, } \\
\text { Israel(19) }\end{array}$ & 1076 & 1993 & $1-18$ & $\begin{array}{l}\text { Diagnosed as } \\
\text { having an acute } \\
\text { asthma attack }\end{array}$ & $\begin{array}{l}\text { Daily Min } \\
\text { and Max } \\
\text { Temperature }\end{array}$ & Ecologic & $C$ & Male & $N R$ & $\begin{array}{c}\text { Barometric } \\
\text { Pressure, Relative } \\
\text { Humidity, Air } \\
\text { pollutant }\end{array}$ & $\begin{array}{l}\text { Pearson } \\
\text { Correlation }\end{array}$ & $\begin{array}{l}\text { ER visits showed a negative } \\
\text { correlation with minimal } \\
\text { temperatures }(r=-0.45) \text { and } \\
\text { maximal temperatures }(r=-0.41)\end{array}$ \\
\hline 17 & $\begin{array}{l}\text { Ivey,2001, } \\
\text { Trinidad and } \\
\text { Tobago(21) }\end{array}$ & 27848 & 1997 & $<16$ & $\begin{array}{l}\text { Diagnosis of } \\
\text { acute asthma } \\
\text { and received } \\
\text { bronchodilator } \\
\text { nebulization }\end{array}$ & $\begin{array}{l}\text { Daily Mean } \\
\text { Temperature }\end{array}$ & $\begin{array}{l}\text { Retrospective } \\
\text { Ecologic Study }\end{array}$ & A & Equal & $N R$ & $\begin{array}{l}\text { Rainfall, Relative } \\
\text { Humidity, Sex }\end{array}$ & $\begin{array}{l}\text { Multiple } \\
\text { Regression }\end{array}$ & $\begin{array}{l}\text { Increased asthma visits in children } \\
\text { were associated with increased } \\
\text { temperature } \beta=0.14270, P=0.009\end{array}$ \\
\hline 18 & $\begin{array}{l}\text { Li,2016, } \\
\text { China(32) }\end{array}$ & 17022 & $\begin{array}{l}2007- \\
2013\end{array}$ & $0-14$ & $\begin{array}{l}\text { Diagnosis by } \\
\text { clinicians }\end{array}$ & $\begin{array}{l}\text { Daily Min, Max } \\
\text { and Mean } \\
\text { Temperature } \\
\text { and } \text { TVN }^{*}\end{array}$ & Ecologic & C & Male & 10 & $\begin{array}{l}\text { Sex, Relative } \\
\text { Humidity }\end{array}$ & $\begin{array}{l}\text { Poisson } \\
\text { Generalized } \\
\text { Linear } \\
\text { Regression }\end{array}$ & $\begin{array}{c}\mathrm{A} 1^{\circ} \mathrm{C} \text { increase in temperature } \\
\text { variation was associated with a } \\
4.2 \%(95 \% \mathrm{Cl} 0.9-7.6 \%) \text { increase } \\
\text { in the number of hospital visits for } \\
\text { childhood asthma }\end{array}$ \\
\hline 19 & $\begin{array}{l}\text { Mireku, } \\
\text { 2009, USA } \\
\text { (36) }\end{array}$ & 25401 & $\begin{array}{l}2004- \\
2005\end{array}$ & $0-18$ & $\begin{array}{l}\text { Diagnosis by } \\
\text { clinicians }\end{array}$ & $\begin{array}{l}\text { Daily Min, Max } \\
\text { and Mean } \\
\text { Temperature }\end{array}$ & $\begin{array}{l}\text { Retrospective } \\
\text { Ecologic Study }\end{array}$ & D & NR & $0-5$ & $\begin{array}{l}\text { Humidity, } \\
\text { Barometric } \\
\text { Pressure, Air } \\
\text { pollutant }\end{array}$ & Time series & $\begin{array}{l}\text { Interday changes in temperature } \\
\text { from } 1 \text { day before asthma attack } \\
\text { increased ED visits, with a } 10^{\circ} \mathrm{F} \\
\text { increase being associated with } 1.8 \\
\text { additional visits }(\mathrm{P}=0.006)\end{array}$ \\
\hline 20 & $\begin{array}{l}\text { O'Lenick, } \\
2017, \\
\text { USA(25) }\end{array}$ & NR & $\begin{array}{l}1993- \\
2012\end{array}$ & $0-19$ & $\begin{array}{l}\text { ICD9-(493.0- } \\
493.9)\end{array}$ & $\begin{array}{l}\text { Daily Min, Max } \\
\text { and Mean } \\
\text { Temperature }\end{array}$ & Ecologic & $C$ & Male & $0-7$ & $\begin{array}{c}\text { Sex, Race, } \\
\text { Insurance Status }\end{array}$ & $\begin{array}{c}\text { Poisson } \\
\text { generalized } \\
\text { linear models }\end{array}$ & $\begin{array}{l}\text { Estimated RRs for T max and } \\
\text { pediatric asthma ED visits were } \\
\text { positive and significant for lag days } \\
1-5, \text { with the strongest single-day } \\
\text { association observed on lag day } 2 \\
\text { (RR=1.06, 95\% Cl: } 1.03,1.09)\end{array}$ \\
\hline 21 & $\begin{array}{l}\text { Zaninovic, } \\
\text { 2001, Croatia } \\
\text { (29) }\end{array}$ & 84 & 1984 & $\begin{array}{l}\text { Not } \\
\text { Reported }\end{array}$ & Not Reported & $\begin{array}{l}\text { Daily Min, Max } \\
\text { and Mean } \\
\text { Temperature }\end{array}$ & Ecologic & C & NR & $0-7$ & $\begin{array}{l}\text { Humidity, } \\
\text { Barometric } \\
\text { Pressure and } \\
\text { Wind Speed }\end{array}$ & $\begin{array}{l}\text { Spearman } \\
\text { Correlation }\end{array}$ & $\begin{array}{l}\text { Negative correlation coefficients } \\
\text { between asthmatic attacks and } \\
\text { mean, maximum and minimum air } \\
\text { temperatures appeared on most days. } \\
\text { The results considered together } \\
\text { point at cold, clear and dry winter } \\
\text { anticyclonic situations as dangerous } \\
\text { for the asthmatics }\end{array}$ \\
\hline 22 & $\begin{array}{l}\text { Palusci, } \\
\text { 1998, USA } \\
\quad(26)\end{array}$ & 6741 & $\begin{array}{l}1991- \\
1995\end{array}$ & $<18$ & Not Reported & $\begin{array}{l}\text { Monthly Min, } \\
\text { Max and Mean } \\
\text { Temperature }\end{array}$ & $\begin{array}{l}\text { Historical } \\
\text { Cohort }\end{array}$ & C & NR & NR & \begin{tabular}{|l|} 
Dew point, \\
Relative Humidity, \\
Barometric \\
Pressure and \\
Wind Speed \\
\end{tabular} & $\begin{array}{l}\text { Multiple } \\
\text { Regression }\end{array}$ & $\begin{array}{l}\text { No effect was seen with an average } \\
\text { temperature }\end{array}$ \\
\hline 23 & $\begin{array}{l}\text { Witonsky, } \\
\text { 2018, } \\
\text { USA(28) }\end{array}$ & 43729 & $\begin{array}{l}2001- \\
2008\end{array}$ & $\begin{array}{l}\text { All age } \\
\text { groups }\end{array}$ & ICD9-(493.00) & $\begin{array}{l}\text { Weekly Mean } \\
\text { Temperature }\end{array}$ & Ecologic & $C$ & NR & NR & $\begin{array}{c}\text { NO2,O3,PM2.5, } \\
\text { SO2, recipitation, } \\
\text { air pressure, } \\
\text { humidity, tree } \\
\text { pollen, grass } \\
\text { pollen, and weed } \\
\text { pollen }\end{array}$ & $\begin{array}{l}\text { Spearman } \\
\text { Correlation }\end{array}$ & $\begin{array}{l}\text { In pediatric patients, the multivariate } \\
\text { coefficients for temperature } \\
\text { and asthma related emergency } \\
\text { department visits in the full year was } \\
-0.351 \text { and in fall was }-0.335\end{array}$ \\
\hline
\end{tabular}

* TVN: Temperature variation between neighboring days

*NR: Not Reported 
outcome in different articles. Although all studies were conducted after the release of the Ninth Revision of the International Classification of Diseases, only 8 studies had used ICD 9 4, 16, 25, 27, 28, 30,31, 34. Five studies had used code 493.00 and 3 studies had used code 493.00 to 493.99 . Also, two studies had used ICD10- (J45) as the outcome variable $^{17,35}$. The rest of the studies measured outcome based on physicians' assessment, the presence of asthmarelated symptoms and the administration of nebulized bronchodilators. Two studies did not report how the asthmatic attack was diagnosed ${ }^{26,29}$.

The results of 10 studies showed that the number of cases of asthma exacerbation was higher in boys than girls, while two studies reported a higher number of cases of asthma in girls than boys ${ }^{4,27}$, and one study reported an equal number of cases $^{36}$.

Studies had considered different lag periods between exposure and outcome, which varied from 0 to 60 days,

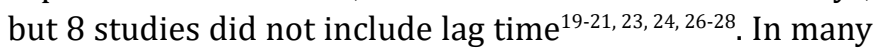
studies, the temperature was not the only risk factor considered; and demographic factors such as sex, race and meteorological factors such as barometric pressure, relative humidity, wind speed, rainfall and air pollutants, such as $\mathrm{PM}_{10}, \mathrm{PM}_{2.5}, \mathrm{O}_{3}, \mathrm{NO}_{\mathrm{X}}$, were also examined. But these variables were different in different studies.

Fourteen studies were simple ecological studies, and two were case-crossover studies ${ }^{27,34}$. Two studies had used the time-series method ${ }^{24,31}$. Seven studies had used correlation coefficients ${ }^{16,17,19,20,24,28,29}$ and four studies had used relative risks to examine the relation between air temperature and asthma exacerbations ${ }^{12,25,27,31}$.

Fourteen studies had reported a relation between cold temperature and recurrence of asthma and showed as the temperature dropped, the number of asthma attacks or the number of visits to hospitals for asthma increased in children $12,16,17,19,20,24,28-35$. Nine papers observed a relation between hot weather and asthma attacks 4 , 18, 21, 22, 25, 31, 32, 35, ${ }^{36}$, and 3 studies reported a relation between temperature differences and asthma attacks ${ }^{13,18,34}$. Two studies did not show any relation between asthma attacks and temperature variables ${ }^{26,27}$.

Studies in Malta and Spain showed that the increase in the incidence of asthma in the 5-14 age group was associated with the school re-opening season, and with a two-week lag after the start of the school year, recurrence of asthma increased in this age group ${ }^{19,20,22,30}$.

Among the papers reviewed, 11 papers had examined the effect of seasons on the recurrence of asthma in children. Based on the Köppen-Geiger Climate Classification 16 studies were conducted in a warm-temperate climate ${ }^{4}$ $12,16,19,20,23-26,29-35$. Three articles were from the equatorial region $^{12,25,34}$ and three articles were from the snow region ${ }^{13,}$
18, 37. Study results were different, but mainly the highest frequency of asthma occurred in autumn and spring ${ }^{4,16,20 \text {, }}$ $22,24,26,28,30,34-36$.

According to the World Economic Situation and Prospects ${ }^{38}$, most studies (16 studies) had been conducted in developed countries; including Croatia, the US, Japan, Australia, Greece, Malta and Spain; and seven studies had been conducted in developing countries, including China, Trinidad and Tobago, Hong Kong, Israel and Brazil ${ }^{17,}$ 19, 21-23, 31,32 , while no study had been conducted in less developed countries.

\section{Discussion}

Most of the studies showed that extreme ambient air temperature has a relation with the recurrence or hospitalization of children due to asthma. For example, in Tokyo, as temperature decreased ${ }^{12}$, and in New York, as temperature increased, the incidence of asthma attacks in children increased ${ }^{4}$. The average daily temperature in Tokyo is $11^{\circ} \mathrm{C}$, while in New York City during cold spells it is $-15{ }^{\circ} \mathrm{C}^{4}$ and New York has colder winters in comparison to Tokyo. Probably children with asthma during the winter season and during the cold spell, because of medical orders, changed their behavior and were less likely to expose themselves to the cold environment or use a mask to prevent asthma attacks.

Cold and hot temperatures affect the respiratory system through different mechanisms. Warm weather may cause microorganisms, mites and cockroaches to grow in the interior of human dwellings, or warm air can cause increased air pollution such as an increase in ozone and $\mathrm{PM}_{10}$. This, maybe one of the factors associated with inflammation of the respiratory tract and the recurrence of asthma.

Studies have also shown that cold and dry air increases the risk of airway inflammation, reduces lung function and reduces lung capacity ${ }^{37,39-41}$. Cold weather can decrease moisture in the mucosal membrane of the respiratory tract, predispose it to irritation by allergens, cause sensitivity to viral and bacterial infections and increase the risk of asthma attacks ${ }^{42}$.

Studies conducted in Japan and America reported a direct relation between temperature changes, one day

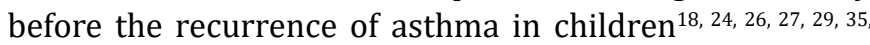
36. But, children under 5 years of age were less likely to be affected by air temperature changes due to less contact with the outside and environmental triggers ${ }^{31,34,35}$.

However, the high recurrence rate of asthma in school age children can be due to temperature changes and the transition from summer to autumn and due to the easier transmission of respiratory diseases after school opens. Respiratory pathogens in these children affect the relation 
between air temperature and the recurrence of asthma. Students can transmit these respiratory diseases to other family members, and a recurrence of asthma may be seen in other age groups as well ${ }^{16,20,22,30}$.

The reason for the difference in recurrence of asthma in different times of the year can be due to the effects of various climatic or demographic variables, air pollutants, viral infections, and indoor aeroallergens ${ }^{43}$. In spring, the increased incidence of asthma may be related to fungal spores and pollen grains, while in autumn it may be due to rapid changes in temperature ${ }^{44,45}$.

Most of the studies used in this review were ecological studies and one of the limitations of these studies was the assumption that the level of exposure was equal throughout the population and in different social and economic classes; and as well as the amount of time spent outdoors, and the use of air conditioner, heater and humidifiers. Also, in these studies, the reference population of patients visiting the hospitals under investigation was not clear. Only two studies used postal codes to determine the patients location of residence and excluded children who were not living in that area ${ }^{34,36}$.

Another limitation was not using the same patient classification system in the studies, as only 10 studies used the International Classification of Diseases ${ }^{4}, 16,17,25,27,30,31,34$, ${ }^{35}$. Other studies used physician's assessment, the presence of asthma symptoms, or a history of using asthma drugs; for diagnosing asthma attacks. Two studies did not report the criteria used for diagnosing asthma attacks ${ }^{26,29}$.

\section{Conclusion}

Ambient air temperature is probably related to the recurrence and hospitalization of child asthmatic patients. The results of some studies have shown that extremely hot and cold temperatures both increase the incidence of asthma in children. However, hot and cold temperature cause asthma attacks through different mechanisms.

\section{References}

1. Khamutian R, Najafi F, Soltanian $\mathrm{M}$, et al. The association between air pollution and weather conditions with increase in the number of admissions of asthmatic patients in emergency wards: A case study in Kermanshah. Medical Journal of the Islamic Republic of Iran. 2015; 29(1).

2. World Health Organization. Asthma Fact sheet No 307. 2013. http:// www.who.int/mediacentre/factsheets/fs307/en/ (Updated April 2017).

3. Brzezińska-Pawłowska OE, Rydzewska AD, Łuczyńska M, MajkowskaWojciechowska B, Kowalski ML, Makowska JS. Environmental factors affecting seasonality of ambulance emergency service visits for exacerbations of asthma and COPD. Journal of Asthma. 2016; 53(2): 139-45.

4. Fitzgerald EF, Pantea C, Lin S. Cold Spells and the Risk of Hospitalization for Asthma: New York, USA 1991-2006. Lung. 2014; 192(6): 947-54.

5. Dadbakhsh M, Khanjani N, Bahrampour A, et al. Death from respiratory diseases and temperature in Shiraz, Iran (2006-2011). Int J Biometeorol. 2017; 61(2): 239-46.

6. Khanjani N, Bahrampour A. Temperature and cardiovascular and respiratory mortality in desert climate. A case study of Kerman, Iran. Iranian journal of environmental health science \& engineering. 2013; 10(1): 11.

7. IPCC.Climate Change 2014 Synthesis Report Summary for Policymakers. https://www.ipcc.ch/report/ar5/syr/ (Updated july 2017).

8. IPCC. Summary for Policymakers. In: Stocker TF, Qin D, Plattner G-K, Tignor M, Allen SK, Boschung J, et al., editors. Climate Change 2013: The Physical Science Basis Contribution of Working Group I to the Fifth Assessment Report of the Intergovernmental Panel on Climate Change. Cambridge, United Kingdom and New York, NY, USA: Cambridge University Press; 2013. p. 1-30.

9. Buckley JP, Richardson DB. Seasonal modification of the association between temperature and adult emergency department visits for asthma: A case-crossover study. Environmental Health: A Global Access Science Source. 2012; 11(1).

10. Picado C. Barcelona's asthma epidemics: clinical aspects and intriguing findings. Thorax. 1992; 47(3): 197-200.

11. Silverstein M, Mair Je Fau - Katusic SK, Katusic Sk Fau - Wollan PC, et al. School attendance and school performance: a population-based study of children. (0022-3476 (Print)).

12. Hashimoto $\mathrm{M}$, Fukuda $\mathrm{T}$, Shimizu $\mathrm{T}$, et al. Influence of climate factors on emergency visits for childhood asthma attack. Pediatrics International. 2004; 46(1): 48-52.

13. Abe T, Tokuda $Y$, Ohde $S$, et al. The relationship of short-term air pollution and weather to ED visits for asthma in Japan. The American Journal of Emergency Medicine. 2009; 27(2): 153-9.

14. Anderson GB, Dominici F, Wang Y, et al. Heat-related Emergency Hospitalizations for Respiratory Diseases in the Medicare Population. Am J Respir Crit Care Med. 2013; 187(10): 1098-103.

15. Xu Z, Crooks JL, Davies JM, et al. The association between ambient temperature and childhood asthma: a systematic review. International journal of biometeorology. 2017; 1-11.

16. Altzibar JM, Tamayo-Uria I, De Castro V, et al. Epidemiology of asthma exacerbations and their relation with environmental factors in the Basque Country. Clinical and experimental allergy : journal of the British Society for Allergy and Clinical Immunology. 2015; 45(6): 1099-108.

17. de Souza A, Santos DAS, Lima APS, et al. Impact of changes in meteorological and hospitalizations for asthma. Espacios. 2015; $36(11)$

18. Ehara A, Takasaki H, Takeda Y, et al. Are high barometric pressure, low humidity and diurnal change of temperature related to the onset of asthmatic symptoms? Pediatrics international : official journal of the Japan Pediatric Society. 2000; 42(3): 272-4.

19. Garty BZ, Kosman E, Ganor E, et al. Emergency Room Visits of Asthmatic Children, Relation to Air Pollution, Weather, and Airborne Allergens. Annals of Allergy, Asthma \& Immunology. 1998; 81(6): 563-70.

20. Grech V, Balzan M, Asciak RP, et al. Seasonal variations in hospital admissions for asthma in Malta. The Journal of asthma : official journal of the Association for the Care of Asthma. 2002; 39(3): 263-8.

21. Ivey MA, Simeon DT, Juman S, et al. Associations between climate variables and asthma visits to accident and emergency facilities in Trinidad, West Indies. Allergology International. 2001; 50(1): 29-33.

22. Ivey MA, Simeon DT, Monteil MA. Climatic variables are associated with seasonal acute asthma admissions to accident and emergency room facilities in Trinidad, West Indies. Clinical and experimental 
allergy : journal of the British Society for Allergy and Clinical Immunology. 2003; 33(11): 1526-30.

23. Liu XM, Zhou XB, Shuang L, et al. Relations between meteorological factors and asthma in children from Qingdao city. Journal of Clinical Rehabilitative Tissue Engineering Research. 2007; 11(16): 3181-3.

24. Nastos PT, Paliatsos AG, Priftis KN, et al. The effect of weather types on the frequency of childhood asthma admissions in Athens, Greece. Fresenius Environmental Bulletin. 2006; 15(8 B): 936-42.

25. O'Lenick CR, Winquist A, Chang HH, et al. Evaluation of individual and area-level factors as modifiers of the association between warmseason temperature and pediatric asthma morbidity in Atlanta, GA. Environmental Research. 2017; 156: 132-44.

26. Palusci VJ, Mustalish EK, Fine J, et al. Does the weather trigger pediatric asthma emergency department visits? Ambulatory Child Health. 1998; 3(4): 357-63.

27. Wasilevich EA, Rabito F, Lefante J, et al. Short-term outdoor temperature change and emergency department visits for asthma among children: a case-crossover study. American journal of epidemiology. 2012; 176 Suppl 7: S123-30.

28. Witonsky J, Abraham R, Toh J, et al. The association of environmental meteorological, and pollen count variables with asthma-related emergency department visits and hospitalizations in the Bronx. Journal of Asthma. 2018.

29. Zaninović K, Raos M. The influence of meteorological parameters on asthmatic attacks in children. Hrvatski Meteoroloski Casopis. 2001; (37): 89-94.

30. Hervas D, Utrera JF, Hervas-Masip J, et al. Can meteorological factors forecast asthma exacerbation in a paediatric population? Allergologia et immunopathologia. 2015; 43(1): 32-6.

31. Lam HCY, Li AM, Chan EYY, et al. The short-term association between asthma hospitalisations, ambient temperature, other meteorological factors and air pollutants in Hong Kong: A time-series study. Thorax. 2016; 71(12): 1097-109.

32. Li K, Ni H, Yang Z, et al. Effects of temperature variation between neighbouring days on daily hospital visits for childhood asthma: a time-series analysis. Public Health. 2016; 136: 133-40.

33. Nastos PT, Paliatsos AG, Papadopoulos M, et al. The effect of weather variability on pediatric asthma admissions in Athens, Greece. The Journal of asthma : official journal of the Association for the Care of Asthma. 2008; 45(1): 59-65.
34. Soneja S, Jiang C, Fisher J, et al. Exposure to extreme heat and precipitation events associated with increased risk of hospitalization for asthma in Maryland, U.S.A. Environmental Health: A Global Access Science Source. 2016; 15(1).

35. Xu ZW, Huang CR, Hu WB, et al. Extreme temperatures and emergency department admissions for childhood asthma in Brisbane, Australia. Occupational and Environmental Medicine. 2013; 70(10): 730-5.

36. Mireku N, Wang Y, Ager J, et al. Changes in weather and the effects on pediatric asthma exacerbations. Annals of Allergy, Asthma \& Immunology. 2009; 103(3): 220-4.

37. Bartoli ML, Vagaggini B, Malagrinò L, et al. Baseline airway inflammation may be a determinant of the response to ozone exposure in asthmatic patients. Inhal Toxicol. 2013; 25(3): 127-33.

38. World Economic Situation and Prospects 2016. http://www.un.org/ en/development/desa/policy/wesp/wesp_current/2014wesp_ country_classification.pdf. United Nations publication.

39. Bartoli ML, Vagaggini B, Malagrinò L, et al. Baseline airway inflammation may be a determinant of ozone response in asthmatic patients. Eur Respiratory Soc; 2012.

40. Scannell C, Chen L, Aris RM, et al. Greater ozone-induced inflammatory responses in subjects with asthma. Am J Respir Crit Care Med. 1996; 154(1): 24-9.

41. Weinmayr G, Romeo E, De Sario M, et al. Short-term effects of PM10 and NO2 on respiratory health among children with asthma or asthma-like symptoms: a systematic review and meta-analysis. Environ Health Perspect. 2010; 118(4): 449

42. Koskela H, Tukiainen $\mathrm{H}$, Kononoff A, et al. Effect of whole-body exposure to cold and wind on lung function in asthmatic patients. Chest. 1994; 105(6): 1728-31.

43. Escamilla-Nuñez MC, Barraza-Villarreal A, Hernandez-Cadena L, et al. Traffic-related air pollution and respiratory symptoms among asthmatic children, resident in Mexico City: the EVA cohort study. Respiratory Research. 2008; 9(1): 74.

44. Johnston NW, Johnston SL, Duncan JM, et al. The September epidemic of asthma exacerbations in children: a search for etiology. Journal of Allergy and Clinical Immunology. 2005; 115(1): 132-8.

45. Berktas B, Bircan A. Effects of atmospheric sulphur dioxide and particulate matter concentrations on emergency room admissions due to asthma in Ankara. Tuberk Toraks. 2003; 51(3): 231-8. 